\title{
Group Contests with Internal Conflict and Power Asymmetry*
}

\author{
Jay Pil Choi ${ }^{\dagger} \quad$ Subhasish M. Chowdhury ${ }^{\ddagger} \quad$ Jaesoo Kim $^{\S}$
}

December 03, 2014

\begin{abstract}
We investigate situations in which players make costly contributions as group members in a group conflict, and at the same time engage in contest with fellow group members to appropriate the possible reward. We introduce within group power asymmetry and complementarity in members' efforts, and analyze how each group's internal conflict influences its chance of winning in the external conflict. We find that a more symmetric group may expend more effort in external conflict when the (common) collective action technology exhibits a high degree of complementarity. Furthermore, depending on the degree of complementarity, the stronger player's relative contribution to external conflict may be higher in a more asymmetric group and, as a result, it is possible for the weaker player to earn a higher payoff. In absence of any complementarity, the rent-dissipation is non-monotonic with the within-group power asymmetry.
\end{abstract}

JEL Classification: C72; D72; D74; H41

Keywords: Conflict; Collective action; Group contest; Asymmetry

${ }^{*}$ We appreciate the useful comments from the editor, two anonymous referees, Kyung Hwan Baik, Martin Kolmar, Karl Warneryd and the participants at the Cesifo Area Conference on Public Sector Economics, the Young Researchers Workshop on Contests and Tournaments at the University of Magdeburg, the XXth Annual Conference of Jadavpur University, the conference on Tournaments, Contests and Relative Performance Evaluation at North Carolina State University, WZB conference on Alliances and Alliance Formation in Conflict, 2012 Annual Conference of the Scottish Economic Association, and the seminar participants at the University of East Anglia and Sungkyunkwan University. Any remaining errors are our own.

${ }^{\dagger}$ School of Economics, University of New South Wales, Sydney, NSW 2052, Australia, Department of Economics, Michigan State University and School of Economics, Yonsei University. E-mail: choijay@msu.edu.

${ }^{\ddagger}$ Corresponding author. School of Economics, Centre for Behavioural and Experimental Social Science, and Centre for Competition Policy, University of East Anglia, Earlham Road, Norwich NR4 7TJ, UK. Phone: +44 160-359-2099. E-mail: s.modak-chowdhury@uea.ac.uk

${ }^{\S}$ Department of Economics, Cavanaugh Hall 511A, 425 University Boulevard, IUPUI, Indianapolis, IN 46202. Phone: +1 317-274-2217. Email: jaeskim@iupui.edu 


\section{Introduction}

Groups often engage in costly confrontations in order to win a reward, while at the same time the group members contest with each other in order to divide the possible reward among themselves. In such cases each group member often makes decision about exerting costly effort for the group collective action, and also about exerting costly effort to compete with own group members. In this article, we study such situations in the shadow of power asymmetry among group members.

There are numerous examples from Political Economy. In an open-list electoral system, a candidate expends resources to convince voters to vote for his party, and separately, to choose himself as the candidate within his party (Ames, 1995). Interest groups compete for rents from government policies while individuals - with possible unequal powers - within the interest group contest for the spoils of the victory (Münster, 2007). When countries in an alliance engage in conflict against another alliance, they also need to decide how to share the burden of costs. This logic works in the same way for the parties within a political alliance (Konrad and Kovenock, 2009). It is also related to the long unanswered issue of the 'linkage between internal and external conflict' in Political Science, and it is noted that "While a variety of theoretical perspectives would argue for such linkages between internal and external conflict, difficult questions continue to focus scholarly attention on this relationship" (Starr, 1994).

Similar examples can be drawn from Industrial and Organizational Economics. Firms producing a system good as complements compete against another system and also divide

profits among themselves. Competing research joint ventures face the same issue. Employees in an organization expend efforts collectively to overcome rival organizations, but at the same time they compete with each other for promotions, bonuses and internal rents (Glazer, 2002). Partners in an organization may compete with each other, as well as with outsider owners to appropriate surplus (Müller and Wärneryd, 2001). Labor unions simultaneously confront 
the authority as well as other unions. But even within a union, workers sharing the same political interest may conflict upon ethnic issues (Dasgupta, 2009). ${ }^{1}$

In all these examples, the nature of internal conflict simultaneously characterizes the shape of external conflict, in particular, through collective action between players within a group. ${ }^{2}$ This article analyzes how the inter-group conflict interacts with intra-group conflict in these environments with special emphases on the power asymmetry between the group members and complementarity in collective action. In a model of group contest, in which players expend resources to both internal and external contest simultaneously and incur additive cost, we use a stochastic (Tullock, 1980) contest success function, consider heterogeneity among group members in terms of power asymmetry, and introduce complementarity in collective action through a Constant Elasticity of Substitution (CES) group impact function. ${ }^{3}$ Consequently, the interplay between the internal and the external conflict turns out to be a key feature in the analysis of the overall contest.

There are two contrasting conventional wisdoms about the question of how each group's internal conflict influences its chance of winning in external conflict. One view suggests that a group with less internal conflict has an advantage in external conflict against a rival group (Deutsch, 1949). The other view is that intra-group conflict is more conducive in eliciting efforts from group members for external conflict (Lüschen, 1970). The group contest literature, in general, does not provide a definitive answer.

Most analyses in the group contest literature focus on issues related to contest design that manipulates total rent dissipation by observing different impact functions, cost structures and value distributions. This area of literature originated with Katz et al. (1990), who

\footnotetext{
${ }^{1}$ Various other important illustrations of such situations come from Biology. Even in nature, different species compete for limited resources within and between species-type simultaneously (Vandermeer, 1975). Sperm competition under polyandry reflects another example of this structure. There are cases in which sperms from a male bird compete with each other in fertilizing the ovum of a polyandric female bird, but at the same time the sperms extract enzymes or take other collective actions that damage (at least the likelihood of the success of) sperms of other males (Baker, 1996; Buckland-Nicks, 1998).

${ }^{2}$ This area of literature dates back to Olson (1965) and was later developed by Becker (1983), Palfrey and Rosenthal (1983), Hardin (1995) among others. This can also be interpreted as the collective action problem in two potentially important environments: competition between groups and internal conflict within a group. See Ostrom (2000) and Sandler and Hartley (2001) for literature reviews.

${ }^{3}$ An impact function is a function that maps group members' individual efforts to the 'group effort' (Wärneryd, 1998). A group contest success function is a function that maps group efforts into the probability of winning the contest (Münster, 2009).
} 
use a perfectly substitutable impact function. The group effort enters in a Tullock contest success function and the winner group is decided. They show that the equilibrium group rent dissipation is unique, however multiple equilibria exist in terms of individual equilibrium efforts. Baik $(1993,2008)$ generalizes the analysis by introducing asymmetric valuation within groups. He shows that the equilibrium rent dissipation by a group depends crucially on the distribution of prize valuation and not on the group size. ${ }^{4}$ Lee (2012) and Chowdhury et al. (2013a) instead use weakest link and best-shot impact functions, respectively. Kolmer and Rommeswinkel (2013) use a CES impact function ranging from weakest link to perfectly substitute. Finally, Chowdhury and Topolyan (2013) analyze cases in which different groups can follow different impact functions. These studies, however, do not model any internal conflict within the groups. ${ }^{5}$

There is an existing area of literature that considers both intra and inter group contest (see, for example, Katz and Tokatlidu, 1996; Wärneryd, 1998; Stein and Rapoport, 2004). However, most of the papers consider a sequence in within and between groups conflicts, i.e., the inter group conflict is assumed to occur before the intra group conflict (or the other way round). Within this framework, important topics such as the effects of introducing an additional level of conflict in fiscal federalism, organizational structure, ownership issues etc. are analyzed. Unlike these studies, Hausken (2005) and Münster (2007) consider the case in which the within and between group contests with Tullock contest success functions occur simultaneously and the players are budget constrained. Hausken (2005) constructs a model with perfectly substitute impact function in which group members expend resources simultaneously to collective action and within group conflict for a fixed prize. He analyzes the effects of group size on equilibrium. He further compares the results with a model in which players expend resources to produce the rent. This concept of allocating scarce resources into production, inter-group conflict and intra-group conflict is later used also by Münster (2007) with a CES impact function. In this model a negative correlation between the intensity

\footnotetext{
${ }^{4}$ A series of follow up analyses include Nitzan (1991), Esteban and Ray (2001), Niou and Tan (2005), Inderst et al. (2008). Please see Konrad (2009) for a survey.

${ }^{5}$ All these studies use a stochastic contest success function. Group contests with deterministic (all-pay auction) contest success function is analyzed by Baik et al. (2001), and Topolyan (2013) for perfectly substitute, Chowdhury et al. (2013b) for weakest link, and Barbieri et al. (2014) for best-shot impact function across groups; and Chowdhury and Topolyan (2014) for heterogenous impact functions across group.
} 
of inter-group conflict and that of intra-group conflict is observed. Münster (2007) further studies the optimal group size and the optimal number of groups from a contest designer's perspective.

Unlike the existing studies, our paper addresses the issue of the impact of the heterogeneity within groups and complementarity on inter-group conflict. At a technical level, we consider linear costs for internal and external conflicts and do not include a budget constraint. Given this, we show that both the views of Deutsch (1949) and Lüschen (1970) have some validity by clarifying the interaction between inter-group and intra-group contests. Furthermore, we ask the following questions. Does internal conflict matter in group members' collective action for external conflict? Since the players are heterogeneous in terms of their within-group power, which player is better off within a group? How significantly can the degree of power asymmetry change the rent dissipation?

The severity of internal conflicts within a group is measured in terms of the rate of rent dissipation within intra-group conflicts. Not surprisingly, as group members have similar power, internal conflict becomes more severe. In this sense, a more conflictive group is defined as one in which the power asymmetry is less. We find (Proposition 1) that a stronger player's relative contribution to the external conflict is higher for the group with a higher degree of power asymmetry. Moreover, a more conflictive group expends more effort in the inter-group conflict, when the (common) collective action technology exhibits a higher degree of complementarity (Proposition 2) . This is because each member's incentive to contribute to the collective action depends on one's equilibrium share of the prize in the internal conflict. Thus, when we compare the weaker individuals within groups, the individual in a more conflictive group is willing to contribute to collective action more than the one in a less conflictive group. The same logic holds for the stronger players. As a result, if individuals' efforts are relatively complementary in impacting the collective action, a more conflictive group faces a free-rider problem (in terms of not expending enough effort in collective action) at a lesser degree.

The answer to the question about whether a stronger player is better off than a weaker player within a group is not straightforward. Although the stronger player can dominate the 
weaker player in internal conflict and have a larger share of the prize, the stronger player usually also contributes more to collective action for external conflict. Hence, when the collective action technology is perfectly complementary, then the stronger player earns a higher payoff than the weaker group member. But, when the technology is perfectly substitute, this results in a non-monotonic relationship between the power asymmetry and the players' payoffs (Lemma 3). A similar outcome (Proposition 4) holds for the relationship between the power asymmetry and the total rent dissipation which is also non-monotonic with the power asymmetry parameter for a perfectly substitute technology and is not maximized when the players are symmetric. This result contrasts starkly to the standard (single) contest models in which the rent dissipation gets smaller with player asymmetry.

The rest of the paper is structured as follows. Section 2 lays out the basic model whereas Section 3 constructs the equilibrium efforts both in inter- and intra-group conflicts. We consider the effects of power asymmetry in external conflict to compare win probabilities in Section 4. In Section 5 and Section 6, we demonstrate the special cases of perfectly substitute and perfectly complementary impact functions to investigate the payoff and rent dissipation. Section 7 concludes.

\section{Model: Collective Action and Conflict Technologies}

Consider two groups, $A$ and $B$, that contest for a reward with common value $R>0$. Each group $G(=A, B)$ consists of two risk-neutral players, $G 1$ and $G 2$. The way the reward is allocated between the two groups depends on the collective efforts put forth by each group. A group's share of the reward is further contested by the members of each group simultaneously. Thus, members of the same group have a common interest and cooperate in external contest against the rival group, but they are competitors against each other in the division of the spoils. Each player chooses two different non-negative efforts: contributing to collective activity for inter-group conflict and contesting a given share of the reward within the group. For the sake of simplicity we assume a linear cost function in which the total cost of effort for a player is the sum of the effort in the internal conflict and the external conflict. Player 
$i$ in group $A(B)$ allocates $a_{i}\left(b_{i}\right)$ units of effort towards internal conflict and $\alpha_{i}\left(\beta_{i}\right)$ units of effort for collective action towards external conflict. We assume complete information and that all players make their decisions simultaneously.

Internal Conflict. Contrary to a substantial part of the literature, we assume players within a group to be heterogeneous by ability or power, where power is defined in terms of advantage conferred in internal conflict. Without any loss of generality, we designate player 1 of each group to have at least as much power as player 2 and thus to have (weak) advantage in internal conflict. This advantage is embedded in the conflict technology. Let $p_{G}\left(x_{1}, x_{2}\right)$ be the probability that player 1 wins in the internal conflict when $x_{1}$ and $x_{2}$ are the internal effort levels exerted by player 1 and 2 ; with $x=a$ when $G=A$, and $x=b$ when $G=B$. Then, internal conflict is resolved by a Tullock (1980) type contest. The contest success function (CSF) in group $G$ is given by

$$
p_{G}\left(x_{1}, x_{2}\right)=\left\{\begin{array}{c}
\frac{x_{1}^{m}}{x_{1}^{m}+\theta_{G} x_{2}^{m}}, \text { if } x_{1}+x_{2} \neq 0 \\
\frac{1}{2}, \text { otherwise. }
\end{array}\right.
$$

where $\theta_{G} \in[0,1]$, and $G=A, B$.

The probability that player 2 wins is simply $1-p_{G}$. The parameter $\theta_{G}$ represents asymmetry in power distribution within group $G$, with a higher $\theta_{G}$ implying a more even power distribution. ${ }^{6}$ For instance, if $\theta_{G}=1$, the power is evenly distributed between the two players, whereas if $\theta_{G}=0$, all the power in internal conflict is possessed by player 1 with $p_{G}\left(x_{1}, x_{2}\right)=1$. We refer to player 1 as the stronger player and player 2 as the weaker player. We also refer to a decrease in $\theta_{G}$ as an increase in the power asymmetry within group $G$.

Collective Action. Let $F\left(y_{1}, y_{2}\right): \boldsymbol{R}_{+}^{2} \rightarrow \boldsymbol{R}_{+}$refer to an impact function that represents collective action of a group in external conflict when the stronger and weaker players contribute $y_{1}$ and $y_{2}$, with $y=\alpha$ when $G=A$, and $y=\beta$ when $G=B$. As discussed

\footnotetext{
${ }^{6}$ One way to interpret this function is that player 1 has some advantage within the group in terms of education, experience, incumbency, technology etc. This specification is introduced by Gradstein (1995). See Skaperdas (1996) and especially Clark and Riis (1998) for axiomatization of this type of contest success function. In this study we focus on Pure Strategy Nash Equilibria, and impose the condition $m \in(0,2)$ to ensure equilibrium in pure strategies.
} 
earlier, in the existing literature, collective action is usually assumed to be a sum of each individual's effort. This assumption of a perfectly substitute impact function ignores any possibility of complementary effects in collective action. However, there are a wide variety of situations in which collective action cannot be treated as the sum of individual members' effort. ${ }^{7}$ We capture the issues of complementarity in collective action by introducing a CES impact function as follows.

$$
F\left(y_{i}, y_{j}\right)=\left[y_{i}^{r}+y_{j}^{r}\right]^{\frac{1}{r}}
$$

From the properties of a CES function, one can note that (i) $F\left(y_{i}, y_{j}\right)$ is concave, $F_{i}\left(y_{i}, y_{j}\right) \geq$ $0, F_{i i}\left(y_{i}, y_{j}\right) \leq 0$, and $F_{i j}\left(y_{i}, y_{j}\right) \geq 0$, where $i, j=1,2$ with $i \neq j$ and the subscripts indicate partial differentiation. Hence, collective action is increasing in each member's contribution, but at a diminishing rate. (ii) This impact function is designed to have a constant returns to scale. (iii) $r \in[-\infty, 1]$ represents the degree of complementarity between individuals' efforts.

External Conflict. The group conflict technology is assumed to be driven by a logit-type contest success function (axiomatized by Münster, 2009). Let $q\left(F\left(\alpha_{1}, \alpha_{2}\right), F\left(\beta_{1}, \beta_{2}\right)\right)$ denote the probability that group $A$ wins in external conflict. Hence:

$$
q\left(F\left(\alpha_{1}, \alpha_{2}\right), F\left(\beta_{1}, \beta_{2}\right)\right)=\left\{\begin{array}{c}
\frac{F\left(\alpha_{1}, \alpha_{2}\right)}{F\left(\alpha_{1}, \alpha_{2}\right)+F\left(\beta_{1}, \beta_{2}\right)}, \text { if } F\left(\alpha_{1}, \alpha_{2}\right)+F\left(\beta_{1}, \beta_{2}\right) \neq 0 \\
\frac{1}{2}, \text { otherwise }
\end{array}\right.
$$

The probability that group $B$ wins is simply $1-q$. To economize on notation, we will often use $\boldsymbol{\alpha}=\left(\alpha_{1}, \alpha_{2}\right)$ and $\boldsymbol{\beta}=\left(\beta_{1}, \beta_{2}\right)$. For instance, $q(\boldsymbol{\alpha}, \boldsymbol{\beta})=\frac{F(\boldsymbol{\alpha})}{F(\boldsymbol{\alpha})+F(\boldsymbol{\beta})}$.

Our formulation assumes that each player makes a decision on his choice of effort in internal and external conflicts simultaneously. It is particularly useful if we interpret $q(\boldsymbol{\alpha}, \boldsymbol{\beta})$ as the probability that group $A$ wins in a winner-take-all external contest. However, if we take the alternative, non-probabilistic, interpretation of $q(\boldsymbol{\alpha}, \boldsymbol{\beta})$ as the share of $A$ 's contested

\footnotetext{
${ }^{7}$ For example, Scully (1995) states "[p]layers interact with one another in team sports. The degree of interaction among player skills determines the nature of the production function." Also, in the early literature of voluntary contributions to a public good, Hirshleifer (1983) studies the possible complementary effect in collective action. Borland (2007) also argues that while the production function in baseball is nearly additive in the sense that hitting and pitching are separate activities, players' efforts are almost perfect complements in American football. See Konrad (2009) chapters 5.5 and 6.3 for detailed discussion in this.
} 
resource, the analyses will still work. In the following we will interpret our results in terms of winner-take-all probabilities in both within and between group contests.

\section{Equilibrium Analysis}

\subsection{Internal Conflict within Groups}

The players in group $A$ maximize the objective functions represented by

$$
\begin{aligned}
V_{A 1} & =p_{A}\left(a_{1}, a_{2}\right) q(\boldsymbol{\alpha}, \boldsymbol{\beta}) R-a_{1}-\alpha_{1} \\
V_{A 2} & =\left[1-p_{A}\left(a_{1}, a_{2}\right)\right] q(\boldsymbol{\alpha}, \boldsymbol{\beta}) R-a_{2}-\alpha_{2} .
\end{aligned}
$$

Similarly, the objective functions for the players in group $B$ are given by

$$
\begin{aligned}
& V_{B 1}=p_{B}\left(b_{1}, b_{2}\right)[1-q(\boldsymbol{\alpha}, \boldsymbol{\beta})] R-b_{1}-\beta_{1} \\
& V_{B 2}=\left[1-p_{B}\left(b_{1}, b_{2}\right)\right][1-q(\boldsymbol{\alpha}, \boldsymbol{\beta})] R-b_{2}-\beta_{2} .
\end{aligned}
$$

We first derive an invariance result that each player's winning probability in their internal conflict $(p, 1-p)$ is independent of the level of their contributions to external conflict $(\boldsymbol{\alpha}, \boldsymbol{\beta})$. The equilibrium probability of winning in internal conflict depends only on the respective group's power distribution parameter $\theta_{G}$. This result, summarized in the following Lemma, considerably simplifies our analysis. ${ }^{8}$

Lemma 1 In equilibrium, both the stronger and the weaker players of group $G$ choose the same level of efforts for internal conflict $\left(a_{1}^{*}=a_{2}^{*}\right.$ and $\left.b_{1}^{*}=b_{2}^{*}\right)$. As a result, the winning probabilities for the stronger and the weaker players depend only on $\theta_{G} ; p_{A}\left(a_{1}^{*}, a_{2}^{*}\right)=\frac{1}{1+\theta_{A}}$ and $p_{B}\left(b_{1}^{*}, b_{2}^{*}\right)=\frac{1}{1+\theta_{B}}$.

\footnotetext{
${ }^{8}$ In this and in the next section we focus on $r \in(-\infty, 1)$ and consider the corner cases of perfectly substitute and perfectly complement in the subsequent sections. Also, following Marchi (2008) we consider only the cases where the Gonzi condition in the payoff functions are satisfied. This ensures both existence, and FOC to be sufficient for the maximization problem. This, however, does not assure uniqueness. As will be shown in the next section, multiple equilibria may exist for symmetric within group power; or for the extreme case of perfect complementary collective technology even for power asymmetry within groups.
} 
To investigate the relationship between the rent dissipation in internal conflict and the power distribution within each group, let us define

$$
\lambda_{A}=\frac{a_{1}^{*}+a_{2}^{*}}{q(\boldsymbol{\alpha}, \boldsymbol{\beta}) R} \text { and } \lambda_{B}=\frac{b_{1}^{*}+b_{2}^{*}}{[1-q(\boldsymbol{\alpha}, \boldsymbol{\beta})] R} .
$$

The denominator of $\lambda_{G}$ represents the expected value of the collective prize for group $G$ in the external conflict whereas the numerator of $\lambda_{G}$ is the total effort expended in internal conflict. Thus, $\lambda_{G}$ is the equilibrium rate of rent dissipation in internal conflict. It measures the level of resources used up for internal conflict relative to the expected value of collective prize for group $G$. The next lemma shows that the group with less power-asymmetry dissipates proportionately more rent out of their expected group prize in internal conflict. In this sense, the group with more even power distribution is more conflictive.

Lemma $2 \quad \lambda_{A} \gtreqless \lambda_{B}$ as $\theta_{A} \gtreqless \theta_{B}$.

Without loss of generality, for the rest of the analyses, we assume $\theta_{A} \leq \theta_{B}$, i.e., the power is more asymmetric in group $A$ compared to group $B$. This implies that group $B$ is more conflictive than group $A$. This, however, does not necessarily mean that players in group $B$ spend more resource for internal conflict. Since the total efforts depend on the size of contestable expected prize, players in group $A$ may expend more efforts in internal conflict if group A's winning probability is much larger in the external conflict.

\subsection{External Conflict between Groups}

Now we study how the inter-group conflict is shaped by the intensity of internal conflict and the distribution of power within each group. With the invariance result from Lemma 1 , we can state each player's objective function in relation to their contribution to external conflict. ${ }^{9}$ For notational simplicity, we denote the equilibrium probability that player 1 wins

\footnotetext{
${ }^{9}$ Casual observation might suggest that the role of power disparity in this model is providing an exogenous division rule of the prize. This is not true because the players' effort levels are important in our comparison of the equilibrium payoffs and the rent-dissipation.
} 
in group $G$ by $p_{G}$. For the members of group $A$, the payoff can be written as follows.

$$
\begin{aligned}
& V_{A 1}=p_{A} q(\boldsymbol{\alpha}, \boldsymbol{\beta}) R-a_{1}^{*}-\alpha_{1} \\
& V_{A 2}=\left(1-p_{A}\right) q(\boldsymbol{\alpha}, \boldsymbol{\beta}) R-a_{2}^{*}-\alpha_{2} .
\end{aligned}
$$

For external conflict, player $i$ in group $A$ maximizes his payoff function $V_{A i}$ by choosing $\alpha_{i}$, where $i=1,2$, given that all players act optimally. One can derive similar conditions for group $B$ members who choose $\beta_{i}$; and the first-order conditions can be expressed as

$$
\begin{aligned}
\frac{F_{1}(\boldsymbol{\alpha}) F(\boldsymbol{\beta})}{[F(\boldsymbol{\alpha})+F(\boldsymbol{\beta})]^{2}} R & =\frac{1}{p_{A}}=\left(1+\theta_{A}\right), \\
\frac{F_{2}(\boldsymbol{\alpha}) F(\boldsymbol{\beta})}{[F(\boldsymbol{\alpha})+F(\boldsymbol{\beta})]^{2}} R & =\frac{1}{1-p_{A}}=\left(\frac{1+\theta_{A}}{\theta_{A}}\right), \\
\frac{F(\boldsymbol{\alpha}) F_{1}(\boldsymbol{\beta})}{[F(\boldsymbol{\alpha})+F(\boldsymbol{\beta})]^{2}} R & =\frac{1}{p_{B}}=\left(1+\theta_{B}\right), \text { and } \\
\frac{F(\boldsymbol{\alpha}) F_{2}(\boldsymbol{\beta})}{[F(\boldsymbol{\alpha})+F(\boldsymbol{\beta})]^{2}} R & =\frac{1}{1-p_{B}}=\left(\frac{1+\theta_{B}}{\theta_{B}}\right) .
\end{aligned}
$$

They can be further manipulated and summarized in the following way.

$$
\begin{aligned}
\frac{F_{1}(\boldsymbol{\alpha})}{F_{2}(\boldsymbol{\alpha})} & =\frac{1-p_{A}}{p_{A}}=\theta_{A}, \\
\frac{F_{1}(\boldsymbol{\beta})}{F_{2}(\boldsymbol{\beta})} & =\frac{1-p_{B}}{p_{B}}=\theta_{B}, \\
\frac{F_{1}(\boldsymbol{\alpha})}{F_{1}(\boldsymbol{\beta})} \frac{F(\boldsymbol{\beta})}{F(\boldsymbol{\alpha})} & =\frac{p_{B}}{p_{A}}=\left(\frac{1+\theta_{A}}{1+\theta_{B}}\right), \text { and } \\
\frac{F_{2}(\boldsymbol{\alpha})}{F_{2}(\boldsymbol{\beta})} \frac{F(\boldsymbol{\beta})}{F(\boldsymbol{\alpha})} & =\frac{1-p_{B}}{1-p_{A}}=\left(\frac{\theta_{B}}{\theta_{A}}\right)\left(\frac{1+\theta_{A}}{1+\theta_{B}}\right)
\end{aligned}
$$

Equations (5) and (6) tell us about the relationship between the marginal contributions of players 1 and 2 in the generation of collective action in each group. In each group, the weaker player's equilibrium marginal contribution to the collective action is greater than the stronger player's. This is because the player with less internal power is expected to receive a smaller share of the prize in external contest. This asymmetry in the relative marginal contributions of the two players translates into the asymmetry in the relative total contributions. Each 
player's incentive to contribute to collective action depends on one's power in the internal conflict. Hence, the relative contribution of player 1 is greater in the less conflictive group $A$. This leads us to the following result.

Proposition 1 The stronger player's relative contribution to external conflict vis-a-vis the weaker player's is higher in group A where power distribution is relatively more asymmetric, i.e., $\quad \frac{\alpha_{1}^{*}}{\alpha_{2}^{*}} \geq \frac{\beta_{1}^{*}}{\beta_{2}^{*}}$ as $\theta_{A} \leq \theta_{B}$ or, $p_{A} \geq p_{B}$.

One important implication of this result is that the two groups exhibit different patterns of inefficiency. Clearly, the generation of collective action in each group is inefficient, because efficiency requires that an individual is compensated with full marginal return of one's effort. It is easy to note that the inefficiency in terms of player 2 (player 1 ) is more pronounced for group $A(B)$ in which the internal power distribution is more asymmetric (symmetric).

\section{Win Probability in External Conflict}

A basic, but unanswered, question is which group has a higher winning probability in external conflict, i.e., whether $q(\boldsymbol{\alpha}, \boldsymbol{\beta})$ is greater than $1 / 2$ or not. This is equivalent to asking whether $\frac{F\left(\alpha_{1}^{*}, \alpha_{2}^{*}\right)}{F\left(\beta_{1}^{*}, \beta_{2}^{*}\right)} \geq 1$. Equations $(7)$ and $(8)$ together result in

$$
\frac{F\left(\alpha_{1}^{*}, \alpha_{2}^{*}\right)}{F\left(\beta_{1}^{*}, \beta_{2}^{*}\right)}=\left(\frac{1+\theta_{B}}{1+\theta_{A}}\right)\left(\frac{\theta_{A}}{\theta_{B}-\theta_{A}}\right)\left[\frac{F_{2}\left(\alpha_{1}^{*}, \alpha_{2}^{*}\right)}{F_{2}\left(\beta_{1}^{*}, \beta_{2}^{*}\right)}-\frac{F_{1}\left(\alpha_{1}^{*}, \alpha_{2}^{*}\right)}{F_{1}\left(\beta_{1}^{*}, \beta_{2}^{*}\right)}\right]
$$

This shows that the answer hinges on the ratio of marginal contributions between stronger and weaker players in equilibrium. Thus, the way in which collective action is generated through individual contributions is crucial to predicting which group will win. In addition, it is worthwhile to study how each group's winning probability is changed by the distribution of power within a group.

An important factor in collective action is a possible complementarity between individual members' contributions. The elasticity of substitution in the CES function is $\frac{1}{1-r}$, which is a measure of the degree of complementarity (or substitutability) between individual members' 
contributions. As $r$ increases, the contributions of the two players in the same group become less complementary (more substitutable). ${ }^{10}$ In the next proposition we derive the relationship between the properties of the group impact function and the group winning probability.

Proposition 2 If the individuals' contributions are relatively complementary in the generation of collective action common to both groups, the winning probability of more conflictive group is greater, and vice versa, i.e., $F\left(\alpha_{1}^{*}, \alpha_{2}^{*}\right) \gtreqless F\left(\beta_{1}^{*}, \beta_{2}^{*}\right)$ as $r \gtreqless 1 / 2$.

At a first sight, the result in Proposition 2 appears to be counter-intuitive. Under circumstances in which collective action requires complementary efforts, the individuals in the more conflictive group contribute to collective action more than in the less conflictive group. Conventional wisdom advises that conflict harms cooperation. However, our result implies that conflict and cooperation can coexist well, in particular, in situations of complementary collective action.

In the case of military alliances, the individuals' contributions are more likely to be substitutable. Thus, our model predicts that leadership in each alliance matters in external conflict. In fact, in the Cold War era, superpower dominance was at issue in the two military alliances, NATO and the Warsaw Pact. On the other hand, in the case of business alliances, the individuals' contributions are more likely to be complementary. For example, the complementarity between the distribution capability and the manufacturing skill is one of the most popular reasons for the strategic alliance. Thus, the size or market power of partners needs to be similar for a strong alliance to form.

A solution for $\frac{F\left(\alpha_{1}^{*}, \alpha_{2}^{*}\right)}{F\left(\beta_{1}^{*}, \beta_{2}^{*}\right)}$ enables us to conduct comparative statics in terms of power distribution to study whether the internal redistribution of power increase or decrease the group's winning probability. One famed argument by Olson (1965) in the context of public goods is that the redistribution of wealth in favor of inequality can make individuals contribute to collective action more, because an individual who gains a significant proportion of total benefits from public goods has more incentive to contribute. We, however, study this issue in terms of

\footnotetext{
${ }^{10}$ While we obtain the most popular, additive impact function with no complementarity as $r$ becomes 1 ; we obtain the impact function with perfect complementarity as $r$ becomes $-\infty$.
} 
power distribution in a group contest. The following Corollary is derived immediately from the Proposition 2.

Corollary The asymmetry of power increases (decreases) the group's probability of winning when the individuals' contributions is relatively substitutable (complementary); i.e., $\frac{\partial}{\partial \theta_{A}}\left(\frac{F\left(\alpha_{1}^{*}, \alpha_{2}^{*}\right)}{F\left(\beta_{1}^{*}, \beta_{2}^{*}\right)}\right) \lesseqgtr 0$ and $\frac{\partial}{\partial \theta_{B}}\left(\frac{F\left(\alpha_{1}^{*}, \alpha_{2}^{*}\right)}{F\left(\beta_{1}^{*}, \beta_{2}^{*}\right)}\right) \gtreqless 0$ as $r \gtreqless 1 / 2$.

This result confirms the intuition of Olson (1965) in a group contest setting. He argues that more asymmetry can facilitate collective action in a public good setting when collective action is defined as the sum of individuals' efforts $(r=1)$. We show that more asymmetry in terms of power can facilitate collective action even in a group contest setting and it is valid for any $r \geq 1 / 2$. In contrast, it should also be emphasized that the result can be sharply reversed if the individuals' contribution is relatively complementary as the case of $r<1 / 2$.

When individuals' efforts are relatively substitutable or the stronger player in the group plays a significant role in the collective action, the redistribution of power towards the stronger player facilitates collective action. This result is consistent with Olson's argument, as the driving force is that the stronger players have more incentives to contribute to collective action. By contrast, this result is sharply reversed for the specific impact functions in which individuals' efforts are relatively complementary or the weaker player turns out to be the crucial player in generating collective action. Thus, in this case, a more equal distribution of power fosters collective action. In addition, the power distribution in a rival group gives the idea of a fierce or a milder conflict and this affects the amount of collective action in a similar way.

To characterize equilibria and comparative statics of the game, one can use Lemma 1 and the results from Kolmar and Rommeswinkel (2013) who fully characterize group contest with CES impact function. We can directly employ a modified version of their result (pp. 12) and find that the effort in the external conflict is independent of the degree of complementarity when there exists no power asymmetry. However, as a simple case, when $\theta_{A}=\theta_{B}=\theta<1$, then in the intermediate range the effort expended by the stronger player will be $\alpha_{1}^{*}=\beta_{1}^{*}=\frac{R}{4(1+\theta)\left[1+(\theta)^{r /(1-r)}\right]}$ and the effort expended by the weaker player will be 
$\alpha_{2}^{*}=\beta_{2}^{*}=\frac{\theta R}{4(1+\theta)\left[1+(1 / \theta)^{r /(1-r)}\right]}$. Note again from the results of Kolmar and Rommeswinkel (2013), however, that for different values of $r$, some cases can only be covered by a limit and the transitions for different values of $r$ are not smooth. It is also possible, as we will show in the next section, to obtain multiple equilibria. As a result in the next section we focus on the two most analyzed, and arguably most interesting polar cases of the $r$ values.

\section{Who is better off: the Stronger or the Weaker Player?}

In addition to the issues discussed in the previous section, an implicit assumption that is made so far to ensure the players to expend strictly positive resources is that the players earn non-negative payoff in the equilibria. This is called a participation constraint. In the standard group contests with only external conflict this constraint is satisfied in equilibrium. However, it may not be so obvious for this particular structure. In this section we focus on two contrasting cases, namely perfectly substitutable (additive effort, when $r=1$ ) and perfectly complementary (weakest link effort, when $r=-\infty$ ) group impact functions, to compare the equilibrium effort levels and payoffs and show that equilibrium exists - for which the participation constraint is also satisfied.

In the case of additive effort group impact function, collective action is performed by the sum of individual group members' efforts, i.e., $F\left(y_{i}, y_{j}\right)=y_{i}+y_{j}$. In the case of weakest link group impact function, the minimum effort among individual group members establishes the level of collective action, i.e., $F\left(y_{i}, y_{j}\right)=\min \left\{y_{i}, y_{j}\right\}$. We can compute the equilibrium effort levels in external conflict as follows. The proof of this lemma comes directly from Baik (1993) and Lee (2012) and is not included.

Lemma 3 (1) Suppose $F\left(y_{i}, y_{j}\right)=y_{i}+y_{j}$. In this case, weaker players completely free-ride in contributing to collective action.

$$
\begin{aligned}
\alpha_{1}^{*} & =\frac{p_{A}^{2} p_{B}}{\left[p_{A}+p_{B}\right]^{2}} R=\frac{\theta_{B}}{\left[2+\theta_{B}+\theta_{B}\right]^{2}} R \geq \\
\frac{p_{A} p_{B}^{2}}{\left[p_{A}+p_{B}\right]^{2}} R & =\frac{\theta_{A}}{\left[2+\theta_{B}+\theta_{B}\right]^{2}} R=\beta_{1}^{*} \text { and } \alpha_{2}^{*}=\beta_{2}^{*}=0
\end{aligned}
$$


(2) Suppose $F\left(y_{i}, y_{j}\right)=\min \left\{y_{i}, y_{j}\right\}$. There are multiple equilibria, but we focus on the payoff-dominant outcome and obtain

$$
\begin{aligned}
\alpha_{1}^{*} & =\alpha_{2}^{*}=\frac{\left[1-p_{A}\right]^{2}\left[1-p_{B}\right]}{\left[1-p_{A}+1-p_{B}\right]^{2}} R=\frac{\theta_{A}^{2} \theta_{B}\left(1+\theta_{B}\right)}{\left[2 \theta_{A} \theta_{B}+\theta_{A}+\theta_{B}\right]^{2}} R \\
& \leq \frac{\left[1-p_{A}\right]\left[1-p_{B}\right]^{2}}{\left[1-p_{A}+1-p_{B}\right]^{2}} R=\frac{\theta_{A} \theta_{B}^{2}\left(1+\theta_{A}\right)}{\left[2 \theta_{A} \theta_{B}+\theta_{A}+\theta_{B}\right]^{2}} R=\beta_{1}^{*}=\beta_{2}^{*} .
\end{aligned}
$$

For additive effort, the winning probability in the external conflict depends only on the stronger players' effort. Thus, the less conflictive group's winning probability is always higher, i.e.,

$$
\text { For } F\left(y_{i}, y_{j}\right)=y_{i}+y_{j}, q\left(\boldsymbol{\alpha}^{*}, \boldsymbol{\beta}^{*}\right)=\frac{p_{A}}{p_{A}+p_{B}} \geq 1 / 2
$$

In the case of weakest-link, as is well-known, multiple equilibria emerge. We select the largest matched effort level because it gives the highest equilibrium payoffs to each player in each group and turns out to be the coalition proof equilibrium (Lee, 2012). Then, the collective action is virtually determined by the weaker players, because the stronger players merely make the same effort as much as the weaker players in own group. In this case, the more conflictive group's winning probability is always higher, i.e.,

$$
\text { For } F\left(y_{i}, y_{j}\right)=\min \left\{y_{i}, y_{j}\right\}, q\left(\boldsymbol{\alpha}^{*}, \boldsymbol{\beta}^{*}\right)=\frac{1-p_{A}}{1-p_{A}+1-p_{B}} \leq 1 / 2 \text {. }
$$

In this sense, these two polar cases make our earlier argument in Proposition 2 and the corresponding Corollary even clearer.

Now, let us compare the equilibrium payoffs of the two players within a group. The following Proposition shows that the stronger players earn higher payoff in the weakest link effort case. However, in the case of additive effort, the result is very different.

Proposition 3 In the weakest-link case, $V_{G 1}^{*} \geq V_{G 2}^{*}$ always for $G=A, B$. In contrast, in the additive effort case, we obtain

$$
V_{A 1}^{*} \geq V_{A 2}^{*} \text { and } V_{B 1}^{*} \geq V_{B 2}^{*} \text { only if } \theta_{B} \geq \frac{\theta_{A}^{2}+2 \theta_{A}-1}{\left(1-\theta_{A}\right)} \text { and } \theta_{A} \geq \frac{\theta_{B}^{2}+2 \theta_{B}-1}{\left(1-\theta_{B}\right)} \text {. }
$$




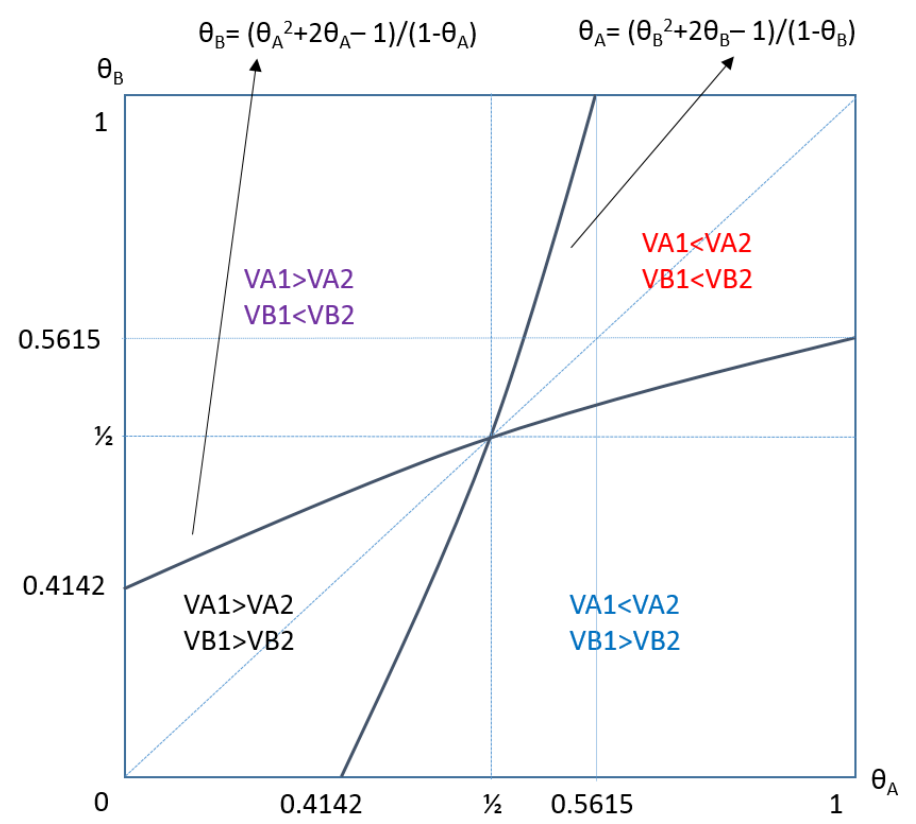

Figure 1. Within-group equilibrium payoff for additive effort

This result is illustrated in Figure 1 . Since we confine our attention to $\theta_{B} \geq \theta_{A}$, we focus on the area above the 45 degree line. Loosely speaking, the result implies that when the power asymmetry among the group members is small enough ( $\theta$ is high enough), then the weaker player has a higher payoff than the stronger player. This is because the weaker player's free riding benefit is large despite his small share of the prize when the asymmetry is small enough. In addition, since the relative benefit of free riding is greater in the more conflictive group, the parameter range in which the weaker player's payoff is greater is larger in the more conflictive group. This becomes completely clear when we consider the polar case, $\theta=\theta_{A}=\theta_{B}$. In this case the result reduces to $V_{G 1}^{*} \gtreqless V_{G 2}^{*}$ as $\theta_{G} \lesseqgtr 1 / 2$.

Getting back to the issue of existence and participation constraint for equilibria, it can be noted that for both the cases showing existence is trivial. Given the effort choices of other players, a player does not deviate from the effort choice outlined in an equilibrium. Furthermore, it is easy to show that the equilibrium payoffs (for the additive case and for the coalition proof weakest link case) of the players are non-negative. Below we provide a 
summary of the result for $\theta=\theta_{A}=\theta_{B}$ and $m=1$ and in the next section we provide a broader discussion regarding rent dissipation.

Example. For the case of weakest-link impact function $F\left(y_{i}, y_{j}\right)=\min \left\{y_{i}, y_{j}\right\}$, we find $a^{*}=\frac{\theta}{(1+\theta)^{2}} \frac{R}{2}$ and $\alpha^{*}=\frac{\theta}{1+\theta} \frac{R}{4}$ from Lemma 1 and Lemma 3 respectively. Each player's symmetric equilibrium payoff is

$$
V=p\left(a^{*}\right) q\left(\alpha^{*}\right) R-a^{*}-\alpha^{*}=\frac{R}{4}\left[1-\frac{3 \theta}{(1+\theta)^{2}}\right] \geq 0
$$

for any $\theta \in[0,1]$. Similarly, for the case of additive impact function $F\left(y_{i}, y_{j}\right)=y_{i}+y_{j}$, we

obtain $a^{*}=\frac{\theta}{(1+\theta)^{2}} \frac{R}{2}$ and $\alpha^{*}=\frac{\theta}{(1+\theta)^{2}} \frac{R}{8}$. Each player's symmetric equilibrium payoff is

$$
V=p\left(a^{*}\right) q\left(\alpha^{*}\right) R-a^{*}-\alpha^{*}=\frac{R}{4}\left[1-\frac{5 \theta}{2(1+\theta)^{2}}\right] \geq 0
$$

for any $\theta \in[0,1]$.

\section{Equilibrium Rent Dissipation}

In this section, we compute the total equilibrium rent dissipation, $\left(a_{1}^{*}+a_{2}^{*}+\alpha_{1}^{*}+\alpha_{2}^{*}\right) / R+$ $\left(b_{1}^{*}+b_{2}^{*}+\beta_{1}^{*}+\beta_{2}^{*}\right) / R$, and analyze how this changes with the power asymmetry. Note that it measures the total efforts relative to the value of the prize, $R$. We have already derived $\alpha_{i}^{*}$ and $\beta_{i}^{*}$ in the previous section, and here we find $a_{i}^{*}$ and $b_{i}^{*}$ for the two impact functions, respectively. Since we are interested in the effect of the power asymmetry on the total rent dissipation across the groups, we now assume symmetric power asymmetry across the groups, i.e., $\theta=\theta_{A}=\theta_{B}$ and $p(\theta)=p_{A}=p_{B}=1 /(1+\theta)$. We first derive the following Lemma.

Lemma 4 Under symmetric power asymmetry across the groups, the rent dissipation in internal conflict is the same in the additive and the weakest link effort cases.

This result allows us to pin down the total rent dissipation for the contest. It is summarized in the next Proposition. 


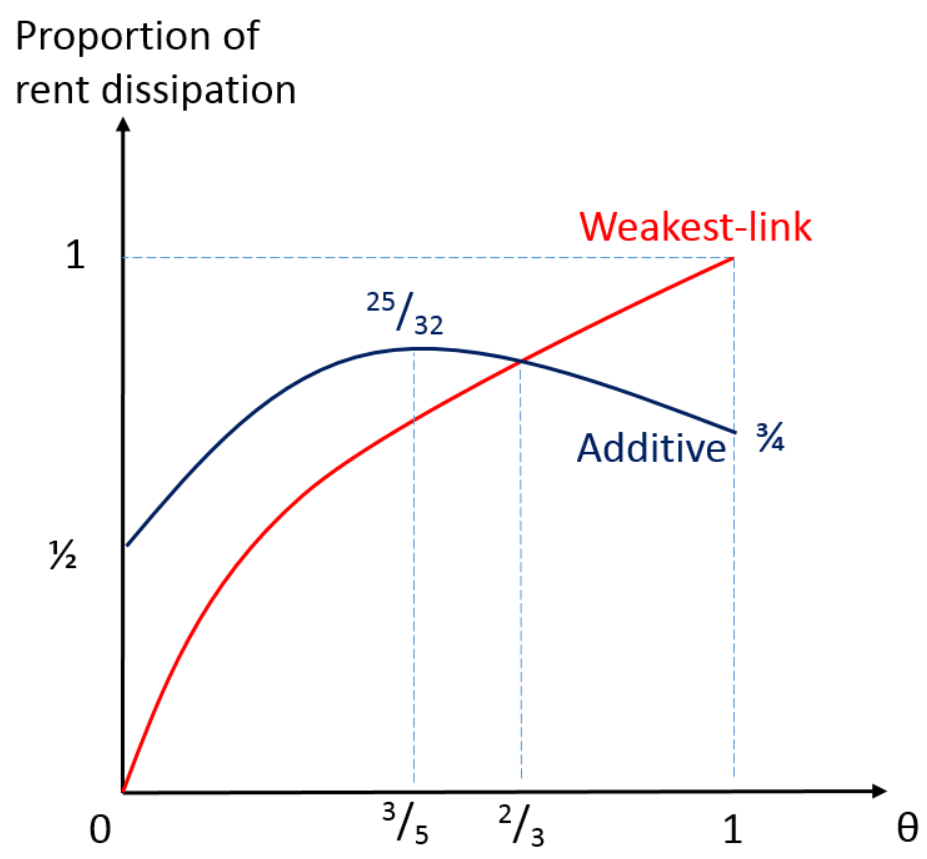

Figure 2. Rent dissipation against power asymmetry

Proposition 4 Under the same power asymmetry across groups, in the weakest-link case, the total rent dissipation is monotonically increasing in $\theta$. In the additive effort case, the total rent dissipation $2 p(\theta)\left(\frac{5}{4}-p(\theta)\right)$ is increasing for $\theta \in[0,3 / 5]$ and decreasing for $\theta \in(3 / 5,1]$.

In the weakest-link case, the rent dissipation is increasing in power asymmetry, i.e., decreasing in $p(\theta)$ both on external and internal conflict. This is observed since when the power asymmetry is smaller, the internal conflict becomes severe. In addition, since the weaker player determines the level of contribution to collective action, the external conflict becomes intense as well. Thus, the total rent dissipation $(2 p(\theta)+1)(1-p(\theta)) R$ is decreasing in $p(\theta)$ (increasing in $\theta$ ).

In contrast, the additive effort case is more interesting. The rent dissipation on internal conflict is obviously decreasing in $p(\theta)$ (increasing in $\theta$ ). However, note that the rent dissipation on external conflict is increasing in $p(\theta)$ (decreasing in $\theta$ ). This means that more severe the external conflict more heterogeneous are the players. It is because the free-rider problem is overshadowed as the stronger player's equilibrium share of the prize is larger. As 
a result, we find that the total rent dissipation does not behave monotonically with the power asymmetry as follows.

This result, represented in Figure 2, has several important implications. First, it is wellknown that the rent dissipation is decreasing in the heterogeneity of the players in a single contest model. This is no longer true in simultaneous inter and intra group contest. Second, our result suggests that the rent dissipation is underestimated in many studies based on the symmetric case single contest. In fact, when the group members are symmetric, the total rent dissipation is not maximized for the additive effort case. Finally, we show that simultaneous contest can be used to ensure full rent dissipation as in the weakest link case. ${ }^{11}$

\section{Discussions}

We develop a model of group contest in which simultaneous within and between group conflict interplay with each other. This structure is an abstract representation of several situations in Political Economy, Industrial and Organizational Economics and Biology. We introduce power asymmetry within groups and complementarity in collective action to analyze their impact on within and inter-group conflicts, equilibrium payoff and rent dissipation. We find that the degree of complementarity plays a crucial role in determining collective action. As a result, a group that faces relatively higher level of inner conflict also contributes more to the between group conflict, only when the degree of complementarity is high. Having a higher power within a group does not necessarily result in higher payoff, when the degree of complementarity is low.

There are interesting ways this analysis can be further pursued in terms of both relaxing some of the assumptions and modifying the structure to incorporate other field applications. As we start our analysis with pre-specified groups, our analysis implicitly suggests that the heterogeneity of individuals will be an important factor in the study of the endogenous formation of groups. It would be an interesting exercise to extend our model to endogenize the group formation problem. Also, in our structure the two groups share the same group

\footnotetext{
${ }^{11}$ One may be interested in the asymmetric case of $\theta_{A}<\theta_{B}$. We also conducted comparative statics of the total rent dissipation with respect to $t<1$ when $\theta_{A}=t \theta_{B}$ and obtained a similar result: the rent dissipation on external competition is increasing in $t$, but that on internal competition is decreasing in $t$.
} 
impact function, it will again be interesting to analyze the situation with different impact functions. Clark and Konrad (2007), and Chowdhury and Topolyan (2013, 2014) study the case where the attackers follow a best-shot and the defenders follow a weakest-link function, respectively. It would be worthwhile to extend their models to be a group contest with internal conflict. Finally, we assume symmetric (unit) marginal cost of internal and external conflict. Relaxing this assumption in terms of either a general non-linear function, or by introducing a budget (as in Hausken, 2005; Münster, 2007; Baik, 2008) may provide different analyses. Several examples discussed in the introduction may also be analyzed by introducing a budget constraint on the total internal and external resources. Those, in our specific structure, may provide some new interesting features. We leave them as avenues for further research. 


\section{References}

[1] Ames, B. (1995). Electoral strategy under open-list proportional representation. American Journal of Political Science, 39(2), 406-433.

[2] Baik, K.H. (1993). Effort Levels in Contests: The Public-Good Prize Case. Economics Letters, 41, 363-67.

[3] Baik, K.H. (2008). Contests with group-specific public-good prizes. Social Choice and Welfare, 30, 103-117.

[4] Baik, K. H., and Lee, S. (2000). Two-stage rent-seeking contests with carryovers. Public Choice, 103, 285-296.

[5] Baker, R. (1996). Sperm wars: The science of sex. New York: Basic Books.

[6] Barbieri, S. and Malueg, D.A., and Topolyan, I. (2014). The Best-shot All-pay (group) Auction with complete information, Economic Theory, 57, 603-640.

[7] Becker, G.S. (1983). A Theory of Competition Among Pressure Groups for Political Influence. Quarterly Journal of Economics, 98:3, 371-400.

[8] Borland, J. (2007). Production Functions for Sporting Teams. Handbook on the Economics of Sport, Edward Elgar.

[9] Buckland-Nicks, J. (1998). Prosobranch parasperm: sterile germ cells that promote paternity? Micron, 29, 267-280.

[10] Chowdhury, S.M., Lee, D., and Sheremeta, R.M. (2013a). Top Guns May Not Fire: Bestshot Group Contests with with Group-Specific Public Good Prize, Journal of Economic Behavior and Organization, 92, 94-103.

[11] Chowdhury, S.M., Lee, D., and Topolyan, I. (2013b). The Max-Min Group Contest, University of East Anglia Working Paper \# 50.

[12] Chowdhury, S.M., and Topolyan, I. (2013). The Attack-and-Defense Group Contests, University of East Anglia Working Paper \# 49. 
[13] Chowdhury, S.M., and Topolyan, I. (2014). The Group All-Pay Auction with Heterogeneous Impact Functions, Mimeo.

[14] Clark, D.J., and Konrad, K.A. (2007). Asymmetric Conflict: weakest-link aginst Best Shot. Journal of Conflict Resolution, 51, 457-469.

[15] Clark, D.J., and Riis, C. (1998). Contest success functions: an extension. Economic Theory, 11, 201- 204.

[16] Dasgupta, I. (2009). , 'Living' wage, class conflict and ethnic strife, Journal of Economic Behavior and Organization, 72, 250-265.

[17] Deutsch, M. (1949). A Theory of Cooperation and Competition. Human Relations, 2, 129-152.

[18] Esteban, J., and Ray, D. (2001). Collective Action and the Group Size Paradox. American Political Science Review, 95(3), 663-672.

[19] Glazer, A. (2002). Allies as rivals: internal and external rent seeking, Journal of Economic Behavior and Organization, 48, 155-162.

[20] Gradstein, M. (1995). Intensity of competition, entry and entry deterrence in rent seeking contests. Economics and Politics, 7, 79-91.

[21] Haig, D., and Bergstrom, C.T. (1995). Multiple mating, sperm competition, and meiotic drive, Journal of Evolutionary Biology, 8, 265-282.

[22] Hardin, R. (1995). One for All: The Logic of Group Conflict. Princeton University Press, Princeton, NJ.

[23] Hausken, K. (2005). Production and Conflict Models Versus Rent-Seeking Models, Public Choice, 123, 59-93.

[24] Hirshleifer, J. (1983). From Weakest-link to Best-shot: The Voluntary Provision of Public Goods. Public Choice, 41(3), 371-386. 
[25] Inderst, R., Müller, H. M., and Wärneryd, K (2006). Distributional conflict in organizations. European Economic Review, 51, 385-402.

[26] Katz, E., Nitzan, S., and Rosenberg, J. (1990). Rent-Seeking for Pure Public Goods. Public Choice, 65(1), 49-60.

[27] Katz, E., and Tokatlidu, J. (1996). Group Competition for Rents. European Journal of Political Economy, 12, 599-607.

[28] Kolmer, M., and Rommeswinkel, H. (2013). Group Contests with Group-specific Public Good and Complementarities in Efforts, Journal of Economic Behavior and Organization, $89,9-22$.

[29] Konrad, K.A. (2009). Strategy and Dynamics in Contests. Oxford University Press.

[30] Konrad, K.A., and Kovenock, D. (2009). The alliance formation puzzle and capacity constraints. Economics Letters, 103, 2, 84-86.

[31] Lee, D. (2012). Weakest-Link Contests with Group-Specific Public Good Prizes. European Journal of Political Economy, 28, 238-248.

[32] Lüschen, G. (1970). Cooperation, Association, and Contest. Journal of Conflict Resolution, 14, 21-34.

[33] Marchi, E. (2008). When is the product of two concave functions concave? University of Minnesota Institute of Mathematics and its Applications Preprint Series \#2204.

[34] Müller, H. M., and Wärneryd, K (2001). Inside versus outside ownership: a political theory of the firm. RAND Journal of Economics, 32(3), 527-541.

[35] Münster, J. (2007). Simultaneous inter- and intra-group conflicts. Economic Theory, 32, $333-352$.

[36] Münster, J. (2009). Group contest success functions. Economic Theory, 41, 345-357.

[37] Niou, E.M., and Tan, G. (2005). External Threat and Collective Action. Economic Inquiry, 43, 519-530. 
[38] Nitzan, S. (1991). Collective Rent Dissipation. Economic Journal, 101, 1522-34.

[39] Olson, M. (1965). The Logic of Collective Action: Public Goods and the Theory of Group. Harvard University Press.

[40] Ostrom, E. (2000). Collective Action and the Evolution of Social Norms. Journal of Economic Perspectives, 14, 37-158.

[41] Palfrey, T. R., and Rosenthal, H. (1983). A Strategic Calculus of Voting. Public Choice, $41,7-53$.

[42] Sandler, T., and Hartley, K. (2001). Economics of Alliances: The Lessons for Collective Action. Journal of Economic Literature, 39, 869-896.

[43] Scully, G. (1995). The Market Structure of Sports. University of Chicago Press.

[44] Skaperdas, S. (1996). Contest Success Functions, Economic Theory, 7, 283-290.

[45] Starr, H. (1994). Revolution and War: Rethinking the Linkage between Internal and External Conflict. Political Research Quarterly, 47, 481-507.

[46] Stein, W. E., and Rapoport, A. (2004). Asymmetric two-stage group rent-seeking: comparison of two contest structures. Public Choice, 118, 151-167.

[47] Topolyan, I. (2013). Rent-seeking for a public good with additive contributions, Social Choice and Welfare, Forthcoming

[48] Tullock, G. (1980). Efficient Rent Seeking. In James M. Buchanan, Robert D. Tollison, Gordon Tullock, (Eds.), Toward a theory of the rent-seeking society. College Station, TX: Texas A\&M University Press, pp. 97-112.

[49] Vandermeer, J. (1975). Interspecific competition: a new approach to the classical theory. Science, 188, 253-255.

[50] Wärneryd, K. (1998). Distributional Conflict and Jurisdictional Organization. Journal of Public Economics, 69, 435-450. 


\section{Appendix}

\subsection{The Proof of Lemma 1.}

The first order conditions with respect to internal conflict in group A are given by

$$
\begin{aligned}
\frac{\partial V_{A 1}}{\partial a_{1}} & =\frac{\theta_{A} m a_{1}^{m-1} a_{2}^{m}}{\left[a_{1}^{m}+\theta_{A} a_{2}^{m}\right]^{2}} q(\boldsymbol{\alpha}, \boldsymbol{\beta}) R-1=0 \\
\frac{\partial V_{A 2}}{\partial a_{2}} & =\frac{\theta_{A} a_{2}^{m-1} a_{1}^{m}}{\left[a_{1}^{m}+\theta_{A} a_{2}^{m}\right]^{2}} q(\boldsymbol{\alpha}, \boldsymbol{\beta}) R-1=0 .
\end{aligned}
$$

The first-order conditions can be summarized by

$$
\frac{a_{1}}{m}=\frac{a_{2}}{m}=\frac{\theta_{A}}{\left(1+\theta_{A}\right)^{2}} q(\boldsymbol{\alpha}, \boldsymbol{\beta}) R
$$

Both the players in group $A$ choose the same level of efforts, $a_{1}^{*}=a_{2}^{*}$ (as functions of $\left.\alpha_{1}, \alpha_{2}, \beta_{1}, \beta_{2}\right)$ for internal conflict regardless of their possibly different choice of $\alpha_{1}$ and $\alpha_{2}$ for external conflict. By proceeding in a similar manner, we can also derive that

$$
\frac{b_{1}}{m}=\frac{b_{2}}{m}=\frac{\theta_{B}}{\left(1+\theta_{B}\right)^{2}}[1-q(\boldsymbol{\alpha}, \boldsymbol{\beta})] R
$$

This implies that $b_{1}^{*}=b_{2}^{*}$. However, the total effort spent on internal conflict can be different for each group. The equilibrium conditions (10) and (11) lead us to the result that the stronger player's winning probabilities in Group $A$ 's internal conflict is $p_{A}\left(a_{1}^{*}, a_{2}^{*}\right)=\frac{1}{1+\theta_{A}}$ and the same for the weaker player is $1-p_{A}\left(a_{1}^{*}, a_{2}^{*}\right)=\frac{\theta_{A}}{1+\theta_{A}}$. A similar result holds for group B internal conflict with $p_{B}\left(b_{1}^{*}, b_{2}^{*}\right)=\frac{1}{1+\theta_{B}}$.

\subsection{The Proof of Proposition 1.}

$F(.,$.$) is a homothetic function. This means that the slopes of the level sets of F(.,$.$) are the$ same along rays coming from the origin. Hence, $\alpha_{1}^{*}\left(\beta_{1}^{*}\right)$ must have a linear relationship with $\alpha_{2}^{*}\left(\beta_{2}^{*}\right)$. Let us define those as $s_{A}=\alpha_{2}^{*} / \alpha_{1}^{*}$ and $s_{B}=\beta_{2}^{*} / \beta_{1}^{*}$. Equations (5) and (6) can then 
be written as

$$
\frac{F_{1}\left(1, \alpha_{2}^{*} / \alpha_{1}^{*}\right)}{F_{2}\left(1, \alpha_{2}^{*} / \alpha_{1}^{*}\right)}=\frac{F_{1}\left(1, s_{A}\right)}{F_{2}\left(1, s_{A}\right)} \leq \frac{F_{1}\left(1, s_{B}\right)}{F_{2}\left(1, s_{B}\right)}=\frac{F_{1}\left(1, \beta_{2}^{*} / \beta_{1}^{*}\right)}{F_{2}\left(1, \beta_{2}^{*} / \beta_{1}^{*}\right)}
$$

because $F_{i}\left(y_{i}, y_{j}\right)$ and $F_{j}\left(y_{i}, y_{j}\right)$ are homogeneous of degree 0 . Note that $\frac{F_{1}\left(1, s_{G}\right)}{F_{2}\left(1, s_{G}\right)}$ is increasing in $s_{G}$ under $F_{j j}\left(y_{i}, y_{j}\right)<0$ and $F_{i j}\left(y_{i}, y_{j}\right)>0$ as follows.

$$
\frac{\partial}{\partial s_{G}}\left[\frac{F_{1}\left(1, s_{G}\right)}{F_{2}\left(1, s_{G}\right)}\right]=\frac{F_{12}\left(1, s_{G}\right) F_{2}\left(1, s_{G}\right)-F_{1}\left(1, s_{G}\right) F_{22}\left(1, s_{G}\right)}{\left[F_{2}\left(1, s_{G}\right)\right]^{2}} \geq 0 .
$$

Therefore we must have $s_{A}=\alpha_{2}^{*} / \alpha_{1}^{*} \leq \beta_{2}^{*} / \beta_{1}^{*}=s_{B}$

\subsection{The Proof of Proposition 2.}

Equation (5), (6), (7), and (8) correspond to

$$
\begin{aligned}
\frac{\alpha_{1}}{\alpha_{2}} & =\left(\frac{1-p_{A}}{p_{A}}\right)^{\frac{1}{r-1}} \\
\frac{\beta_{1}}{\beta_{2}} & =\left(\frac{1-p_{B}}{p_{B}}\right)^{\frac{1}{r-1}} \\
\left(\frac{\alpha_{1}}{\beta_{1}}\right)^{r-1} \frac{\beta_{1}^{r}+\beta_{2}^{r}}{\alpha_{1}^{r}+\alpha_{2}^{r}} & =\frac{p_{B}}{p_{A}} \text { and } \\
\left(\frac{\alpha_{2}}{\beta_{2}}\right)^{r-1} \frac{\beta_{1}^{r}+\beta_{2}^{r}}{\alpha_{1}^{r}+\alpha_{2}^{r}} & =\frac{1-p_{B}}{1-p_{A}} .
\end{aligned}
$$

Define $\rho=\frac{r}{1-r}$. Putting equation (5') and (6') into (7'), we obtain $\left(\frac{\alpha_{1}}{\beta_{1}}\right)=\frac{p_{B}}{p_{A}} \cdot \frac{1+\left(\frac{p_{A}}{1-p_{A}}\right)^{\frac{r}{r-1}}}{1+\left(\frac{p_{B}}{1-p_{B}}\right)^{\frac{r}{r-1}}}$. Plugging this into $\left(7^{\prime}\right)$ again, we get $\frac{\alpha_{1}^{r}+\alpha_{2}^{r}}{\beta_{1}^{r}+\beta_{2}^{r}}=\left(\frac{1+\left(\frac{p_{A}}{1-p_{A}}\right)^{\frac{r}{r-1}}}{1+\left(\frac{p_{B}}{1-p_{B}}\right)^{\frac{r}{r-1}}}\right)^{1-r}\left(\frac{p_{B}}{p_{A}}\right)^{r}$. Using this, we can further manipulate the equation as follows.

$$
\begin{aligned}
\frac{F\left(\alpha_{1}^{*}, \alpha_{2}^{*}\right)}{F\left(\beta_{1}^{*}, \beta_{2}^{*}\right)} & =\left(\frac{\alpha_{1}^{r}+\alpha_{2}^{r}}{\beta_{1}^{r}+\beta_{2}^{r}}\right)^{\frac{1}{r}}=\left(\frac{1+\left(\frac{p_{A}}{1-p_{A}}\right)^{\frac{r}{r-1}}}{1+\left(\frac{p_{B}}{1-p_{B}}\right)^{\frac{r}{r-1}}}\right)^{\frac{1-r}{r}}\left(\frac{p_{A}}{p_{B}}\right) \\
& =\left(\frac{p_{A}^{\frac{r}{1-r}}+\left(1-p_{A}\right)^{\frac{r}{1-r}}}{p_{B}^{\frac{r}{1-r}}+\left(1-p_{B}\right)^{\frac{r}{1-r}}}\right)^{\frac{1-r}{r}}
\end{aligned}
$$


Now, $F\left(\alpha_{1}^{*}, \alpha_{2}^{*}\right) \gtreqless F\left(\beta_{1}^{*}, \beta_{2}^{*}\right)$ is comparable to $p_{A}^{\rho}+\left(1-p_{A}\right)^{\rho} \gtreqless p_{B}^{\rho}+\left(1-p_{B}\right)^{\rho}$. Let us define the function,

$$
g(x)=x^{\rho}+(1-x)^{\rho}, \text { where } x \geq 1 / 2
$$

This function is increasing in $x$ for $\rho>1$ and decreasing in $x$ for $\rho<1$, because $g^{\prime}(x)=$ $\rho\left(x^{\rho-1}-(1-x)^{\rho-1}\right)$. Note that $\rho$ must be greater than 0 for $r<1$. Therefore, since $p\left(\theta_{A}\right)>p\left(\theta_{B}\right), F\left(\alpha_{1}^{*}, \alpha_{2}^{*}\right) \gtreqless F\left(\beta_{1}^{*}, \beta_{2}^{*}\right)$ must correspond to $\rho \gtreqless 1$, which is again equivalent to $r \gtreqless 1 / 2$.

\subsection{The Proof of Proposition 4.}

The difference between the two players' equilibrium payoffs in group A is written as

$$
V_{A 1}^{*}-V_{A 2}^{*}=\left(2 p_{A}-1\right) q(\boldsymbol{\alpha}, \boldsymbol{\beta}) R-\left(\alpha_{1}^{*}+a_{1}^{*}\right)+\left(\alpha_{2}^{*}+a_{2}^{*}\right)
$$

From Lemma 1, both players exert the same level of effort in internal conflict, i.e., $a_{1}^{*}=a_{2}^{*}$. In addition, from Lemma 3, in the case of weakest-link effort, they also contribute the same level of effort to collective action, i.e., $\alpha_{1}^{*}=\alpha_{2}^{*}$. Finally, by construction $\theta_{A}<1$ i.e., $p\left(\theta_{A}\right)>1 / 2$. As a result, we must have $V_{A 1}^{*} \geq V_{A 2}^{*}$. The same logic applies to the players in group B. Hence, the stronger player always has a higher payoff than the weaker player in the weakest-link case.

Applying the results from Lemma 1 and Lemma 3, the difference between the two players' equilibrium payoffs in group A for the additive effort case boils down to

$$
\begin{aligned}
V_{A 1}^{*}-V_{A 2}^{*} & =\left(2 p_{A}-1\right) q(\boldsymbol{\alpha}, \boldsymbol{\beta}) R-\alpha_{1}^{*} \\
& =\frac{p_{A}}{p_{A}+p_{B}} R\left[\left(2 p_{A}-1\right)-\frac{p_{A} p_{B}}{p_{A}+p_{B}}\right]
\end{aligned}
$$

The stronger player's advantage is a higher winning probability in internal conflict. However, the stronger player's disadvantage is that only he has to contribute to external conflict because the weaker player free rides completely, $\alpha_{2}^{*}=0$. As a result, the stronger player has a (weakly) higher payoff than the weaker player in group A in the weakest-link case only if the second 
part of the above given expression in non negative. This condition, after expressing the probabilities in terms of the asymmetry parameter becomes $\theta_{B} \geq \frac{\theta_{A}^{2}+2 \theta_{A}-1}{\left(1-\theta_{A}\right)}$. Similarly, in group B, the stronger player has a (weakly) higher payoff than the weaker player in group A in the weakest-link case only if $\theta_{A} \geq \frac{\theta_{B}{ }^{2}+2 \theta_{B}-1}{\left(1-\theta_{B}\right)}$. Combining these two conditions, we obtain the result

\subsection{The Proof of Lemma 4.}

Inserting $q\left(\boldsymbol{\alpha}^{*}, \boldsymbol{\beta}^{*}\right)$ into (10) and (11), we obtain

$$
\begin{aligned}
& \text { For } F\left(y_{i}, y_{j}\right)=y_{i}+y_{j},\left\{\begin{array}{l}
a_{1}^{*}=a_{2}^{*}=\frac{p_{A}^{2}\left(1-p_{A}\right)}{p_{A}+p_{B}} R \\
b_{1}^{*}=b_{2}^{*}=\frac{p_{B}^{2}\left(1-p_{B}\right)}{p_{A}+p_{B}} R
\end{array}\right. \\
& \text { For } F\left(y_{i}, y_{j}\right)=\min \left\{y_{i}, y_{j}\right\},\left\{\begin{array}{l}
a_{1}^{*}=a_{2}^{*}=\frac{p_{A}\left(1-p_{A}\right)^{2}}{\left(1-p_{A}\right)+\left(1-p_{B}\right)} R \\
b_{1}^{*}=b_{2}^{*}=\frac{p_{B}\left(1-p_{B}\right)^{2}}{\left(1-p_{A}\right)+\left(1-p_{B}\right)} R .
\end{array}\right.
\end{aligned}
$$

Imposing $\theta=\theta_{A}=\theta_{B}$ and $p(\theta)=p_{A}=p_{B}=1 /(1+\theta)$, the total rent dissipation in internal conflict for both the weakest link and the additive effort case turns out to be $\left(a_{1}^{*}+a_{2}^{*}+b_{1}^{*}+b_{2}^{*}\right) / R=2 p(\theta)(1-p(\theta))$

\subsection{The Proof of Proposition 5.}

Using the results of Lemma 4, the following results can be immediately derived.

$$
\begin{aligned}
& \text { For } F\left(y_{i}, y_{j}\right)=y_{i}+y_{j},\left\{\begin{array}{c}
\left(\alpha_{1}^{*}+\alpha_{2}^{*}+\beta_{1}^{*}+\beta_{2}^{*}\right) / R=\frac{p(\theta)}{2} \\
\left(a_{1}^{*}+a_{2}^{*}+b_{1}^{*}+b_{2}^{*}\right) / R=2 p(\theta)(1-p(\theta))
\end{array}\right. \\
& \text { For } F\left(y_{i}, y_{j}\right)=\min \left\{y_{i}, y_{j}\right\},\left\{\begin{array}{c}
\left(\alpha_{1}^{*}+\alpha_{2}^{*}+\beta_{1}^{*}+\beta_{2}^{*}\right) / R=(1-p(\theta)) \\
\left(a_{1}^{*}+a_{2}^{*}+b_{1}^{*}+b_{2}^{*}\right) / R=2 p(\theta)(1-p(\theta)) .
\end{array}\right.
\end{aligned}
$$

In the weakest-link case, The total rent dissipation is: $T R \equiv\left(a_{1}^{*}+a_{2}^{*}+\alpha_{1}^{*}+\alpha_{2}^{*}\right) / R+$ $\left(b_{1}^{*}+b_{2}^{*}+\beta_{1}^{*}+\beta_{2}^{*}\right) / R=(2 p(\theta)+1)(1-p(\theta))=\frac{\theta^{2}+3 \theta}{(1+\theta)^{2}}$. It is easy to show that $\frac{d(T R)}{d \theta}>0$. Hence, total rent dissipation is decreasing in the power asymmetry. In the additive effort 
case $T R \equiv\left(a_{1}^{*}+a_{2}^{*}+\alpha_{1}^{*}+\alpha_{2}^{*}\right) / R+\left(b_{1}^{*}+b_{2}^{*}+\beta_{1}^{*}+\beta_{2}^{*}\right) / R=\left(\frac{5}{2} p(\theta)-2 p(\theta)^{2}\right)=\frac{5 \theta-1}{(1+\theta)^{2}}$. It is easy to show from $\frac{d(T R)}{d \theta}$ that rent dissipation is $1 / 2$ when $\theta=0$, is increasing and reaches its maximum at $\theta=3 / 5$ then it declines to $3 / 4$ when $\theta=1$. 